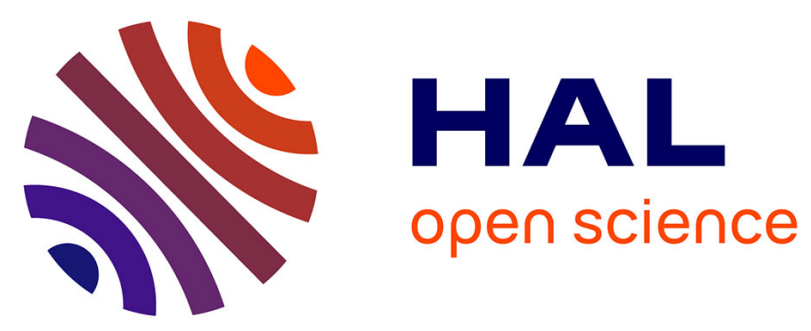

\title{
Construction and analysis of a head CT-scan database for craniofacial reconstruction
}

Françoise Tilotta, Frédéric Richard, Joan Alexis Glaunès, Maxime Berar, Servane Gey, Stéphane Verdeille, Yves Rozenholc, Jean-François F. Gaudy

\section{To cite this version:}

Françoise Tilotta, Frédéric Richard, Joan Alexis Glaunès, Maxime Berar, Servane Gey, et al.. Construction and analysis of a head CT-scan database for craniofacial reconstruction. Forensic Science International, 2009, 191 (1-3), pp.112.e1-112.e12. 10.1016/j.forsciint.2009.06.017 . hal-00278579

HAL Id: hal-00278579

https: / hal-univ-paris.archives-ouvertes.fr/hal-00278579

Submitted on 13 May 2008

HAL is a multi-disciplinary open access archive for the deposit and dissemination of scientific research documents, whether they are published or not. The documents may come from teaching and research institutions in France or abroad, or from public or private research centers.
L'archive ouverte pluridisciplinaire HAL, est destinée au dépôt et à la diffusion de documents scientifiques de niveau recherche, publiés ou non, émanant des établissements d'enseignement et de recherche français ou étrangers, des laboratoires publics ou privés. 


\section{CONSTRUCTION AND ANALYSIS OF A HEAD CT-SCAN DATABASE FOR CRANIOFACIAL RECONSTRUCTION}

Françoise Tilotta (1), Frédéric Richard (2), Joan Glaunès (2), Maxime Berar (2), Servane Gey (2), Stéphane Verdeille (3), Yves Rozenholc (2), Jean F. Gaudy (1).

Author information:

(1) University Paris Descartes

Laboratory of Functional Anatomy

1 rue Marcel Arnoux

92120 Montrouge

France.

(2) University Paris Descartes, MAP5, CNRS UMR 8541

45 rue des Saints Peres

75270 Paris Cedex

France.

(3) Center of Medical Imaging, Val D'or 92210 Saint Cloud

France.

Corresponding author : Frédéric Richard.

frederic.richard@univ-paris5.fr

Tel: 33 (0)1 44553541

Fax: 33 (0)1 42864144

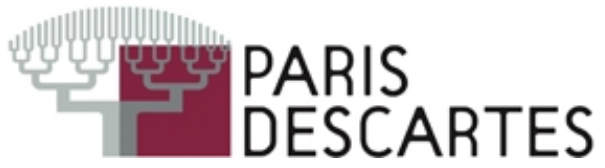

Date: May 13, 2008. 
Abstract. This paper is devoted to the construction of a complete database which is intended to improve the implementation and the evaluation of computerbased facial reconstruction. This growing database is currently composed of 85 head CT-scans of healthy european subjects aged 20 to 65 years old. It also includes the triangulated surfaces of the face and the skull of each subject. These surfaces are extracted from CT-scans using an original combination of imageprocessing techniques which are presented in the paper. Besides, a set of 39 referenced anatomical skull landmarks were located manually on each scan. Using the geometrical information provided by triangulated surfaces, we compute facial soft-tissue depths at each known landmark positions. We report the average thickness values at each landmark and compare our measures to those of the traditional charts of Rhine et al. and of several recent in vivo studies (Manheim et al., De Greef et al. and Helmer et al.).

Keywords: database, head CT-scan, skull segmentation, facial reconstruction, soft-tissue depths

\section{INTRODUCTION}

In forensic medicine, craniofacial reconstruction refers to any process that aims to recover the morphology of a face from observation of a skull. Facial reconstruction is commonly used for the identification of a deceased and non-identified person, when other forensic methods such as dental or DNA analysis do not give satisfactory results. Traditional methods of facial reconstruction use manual techniques such as drawing or sculpture. Sculpture is based on the application of markers representing the thickness of soft tissue at reference landmarks on the craniofacial block [16]. These values are usually taken from tables that are relatively old and which were drawn up by measuring cadavers [35]. Drawing techniques require a certain degree of expertise in graphic art. Both drawing and sculpture therefore are relatively difficult to perform and their major disadvantage lies in the fact that both leave a large part of subjectivity to the artist [17].

In recent years, the development of IT and medical imaging has had a major impact on facial reconstruction (see the surveys in [2, 5, 9, 42] and references therein). New strategies have been proposed which reduce timelines and subjectivity in comparison with earlier reconstruction methods. A computer-based reconstruction is usually obtained by fitting one or several face templates to a dry skull. There are two main fitting approaches: a sparse approach which is based on a limited set of anatomical points (landmarks) located on the face template [3, 11, 38, 44, 45], and a dense approach which is based on a complete template of both the face and the skull [1, 22, 29, 32, 40, 41, 43]. In sparse approaches, the fitting of the face template and the skull is typically obtained in two steps [45]. First, landmarks of the face template are matched to equivalent landmarks on the dry skull, taking into account some estimations of soft tissue depths at landmark positions. Second, the landmark matching is extended to the whole face template using interpolation techniques. In 
dense approaches, the skull template is first mapped onto the dry skull using a registration technique. The deformation obtained from the skull registration is then applied to the face template, leading to an estimation of the skull face.

The template used for a reconstruction is usually derived from a database: it may be an individual selected in a database according to ancestry, age and gender $[32,45]$, an average of several selected individuals [41, 43], or a statistical model obtained from the analysis of a whole database [1,3]. Hence, whatever the definition of templates, performances of a reconstruction technique depend on the quality of the database used. More precisely, they rely upon the ability of the database to represent any skull of a given population.

Databases required for dense and sparse reconstruction techniques are of two different natures. For the implementation of a dense technique, the database must be made of samples where face and skull are both available. For a sparse technique, a database sample can be restricted to a face marked with some landmarks where the soft tissue thickness is measured. Facial images have been acquired using techniques such as laser imagery [45] or ultrasound imagery [3]. Since those acquisition techniques are non-invasive, they make it possible to build up large databases. Skull and face images are best acquired using head CT-scans, which provide good definitions of both structures. However, this imaging technique is invasive and for ethical and legal reasons, it is not only difficult to build up a large database of CT-scans but also to obtain data from healthy subjects. For this reason, the size and quality of CT-scan databases used in dense approaches are usually restricted $[1,32,43]$. Let us however mention the work of Tu et al. [41], who have a private database of 280 subjects from six different origins.

The work presented in this paper is part of a pluridisciplinary project grouping together anatomists, radiologists and mathematicians from University Paris Descartes. The long-term goal of this project is to build and compare facial reconstruction techniques [40]. Here, we focus on the construction of a head CT-scan database of healthy people, which is intended to become the keystone of our project. In order to facilitate the implementation and comparison of facial reconstruction techniques, we plan to give open access to this database for research purposes.

The database we propose is composed of several types of data. First, it contains the head CT-scans of several subjects acquired using a set protocol. In addition to this raw data, it also includes some triangulated and closed surfaces covering the skull and the face of each subject. These surfaces are computed from CT-scans using an original combination of image-processing techniques which are presented in this paper. Lastly, the database includes a reference table of soft tissue measurements at predefined anatomical landmarks of the skull on surfaces extracted from our database.

In Section 2, we describe the acquisition of head CT-scans and provide some statistics concerning the studied population. In Section 3, we present the imageprocessing techniques we designed for the extraction of face and skull surfaces from 
CT-scans. In Section 4, we give and discuss the table of our soft tissue thickness measurements.

\section{Image Database Acquisition}

The study is performed on healthy volunteers using whole head CT scanners (Somatom Sensation 16 - SIEMENS) at the Ouest Parisien (Val d'Or, Saint-Cloud, France) Medical Imaging Center. To comply with the law dated August 9, 2004 relative to public health policy and with the European Directive 2001/20/CE relative to good practices in clinical trial conduct, the research project protocol was submitted to the Paris-Necker Ethics Committee who approved the research on December $5,2005$.

The inclusion criteria retained to build the database are: adult European subjects, males and females, aged 20 to 65 years old. Exclusion criteria were: craniofacial diseases and diseases with craniofacial repercussions, facial asymmetry, past facial trauma (particularly nasal trauma), removeable denture prostheses (partial and complete), dental fixtures and bridges, facial plastic surgery, maxillofacial and orthognatic surgery. Patients having undergone dental or facial orthopedic treatment are included but this data is recorded.

2.1. Composition of the sample. Our database is currently comprised of 85 subjects corresponding to the defined inclusion criteria and whose sex, date of birth, weight, height and the date of any dental or facial orthopedic treatment are known. The long-term goal is to constitute a database of at least 160 subjects distributed according to sex and age group (20 - 40 years and 41 - 65 years). The current distribution of the database is given in Table 1 for age and Table 2 for Body Mass Index.

These age groups are determined so as to take ageing into account. The lower limit for the first age group is 20 years. Since the first external, visible signs of skin ageing appear at about the age of 40 years, we set the upper bound of the first age group at 40 years.

We then set the upper limit of the second age group at 65 years, the age when deeper wrinkles appear and features slacken.

In the framework of this study, we focus on 40 women aged from 20 to 40 years. Our database already contains the data of seven women who are up to five months

older than the age limit selected for our sample population. We include them in the study.

\begin{tabular}{|c|c|c|}
\hline Age (years) & $20-40$ & $41-65$ \\
\hline Male & 17 & 9 \\
\hline Female & 40 & 19 \\
\hline
\end{tabular}

TABLE 1. Distribution of subjects in database according to age and sex. 


\begin{tabular}{|lr|c|}
\hline Type & BMI & N \\
\hline Underweight & $(<19)$ & 8 \\
\hline Normal weight & $(19-24)$ & 34 \\
\hline Overweight & $(24-30)$ & 5 \\
\hline Adiposity & $(30-40)$ & 0 \\
\hline Obesity & $(>40)$ & 0 \\
\hline Total & & 47 \\
\hline
\end{tabular}

TABLE 2. Distribution of subjects in database according to Body Mass Index (BMI)

Our sample size is therefore 47 subjects, all presumed to be healthy.

To ensure the confidentiality required by biomedical research legislation, data is recorded using a code of six letters.

2.2. Subject Positioning. The examination is performed with the subject in the dorsal decubitus position. The subject is placed in occlusion with the tongue against the palate. Acquisition is performed parallel to the Frankfurt plane (or the auriculoorbital plane passing through the two porions and the lowest point on the lower edge of the left orbital cavity).

A preliminary digital image or "scout view" ensures that the subject's head is in the correct position.

2.3. Acquisition parameters. The Dose $\mathrm{x}$ Length product (DLP) refers to the dose of X-rays delivered during the complete procedure. This value accurately represents exposure by relating the dose to the volume being investigated. By taking into account the organs situated within this volume, the Dose $\mathrm{x}$ Length product calculates the effective dose, which is the overall theoretical dose of radiation received by the subject from exposure. In this study, the Dose $\mathrm{x}$ Length product was $1031 \mathrm{mGy} / \mathrm{cm}$, which corresponds to an effective dose of $2.16 \mathrm{mSv}$ if the headspecific conversion factor 0.0021 is used, in compliance with the reference EC99 text. Parameter specifications are shown in Table 3.

2.4. Anatomical Points. Most of the definitions of reference craniofacial landmarks and measurements used in biometrics were standardized by Martin and Saller in 1956 [27]. However, their work was published in German and translation has introduced a certain amount of ambiguity. These definitions were updated by Peyre [30] and Menin [28] at the Laboratory of Vertebrate and Human Paleontology of the Pierre and Marie Curie University. Their work removed some of the inaccuracies caused by translation and proposed some new approaches, including determination of gonion placement. In our study, we use the definitions of these two authors. These landmarks are described in the appendix in Tables 6 and 7.

We choose to keep only those reference landmarks that are easy to locate on scan slices. They are either noteworthy anatomical landmarks or natural borders. They 


\begin{tabular}{|l|l|}
\hline Acquisition parameters & Specifications \\
\hline Pixel matrix & $512 \times 512$ \\
\hline Thickness & $0.75 \mathrm{~mm}$ \\
\hline Pitch & $0.7 \mathrm{~mm}$ \\
\hline Pixel size & 0.48 or $0.529 \mathrm{~mm}$ \\
\hline Reconstruction algorithm & $\mathrm{H} 30 \mathrm{~s}$ \\
\hline Orientation & RAB \\
\hline Acquisition angle & 0 degree \\
\hline Voltage & $120 \mathrm{kV}$ \\
\hline Radiation intensity & $200 \mathrm{~mA}$ \\
\hline Volume CTDI & $42.32 \mathrm{mGy}$ \\
\hline DLP & $1031 \mathrm{mGy} / \mathrm{cm}$ \\
\hline
\end{tabular}

TABLE 3. Specification of acquisition parameters.

may also be constructed from cavities, foraminae or from curve ruptures generated by bony rims. They are shown in Figure 1 .
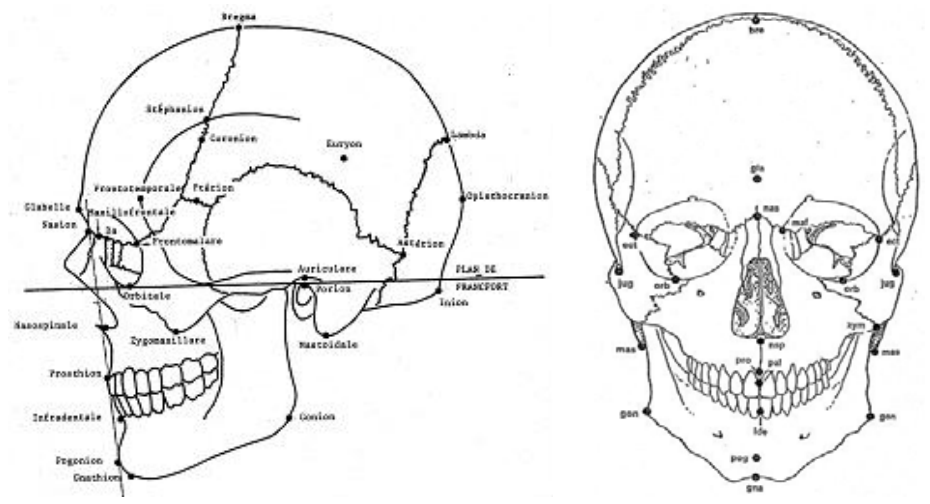

FIGURE 1. Skull anatomical landmarks.

\section{IMAGE PROCESSING}

Here we describe the different techniques we designed to extract two surfaces from a CT-scan:

- bone, which covers the external surface of the skull and wraps the bone areas of the head,

- soft tissue, which wraps soft tissue areas and forms the face.

We also present some tools we used to calculate:

- soft tissue depths on the skull surfaces,

- surface geodesics and patchs. 
The surface extraction technique is divided into four steps. First, we located the bone and soft tissue areas using a simple segmentation technique. Second, using a PDE-driven evolution curve technique, we extracted from each slice two closed curves wrapping soft tissue areas and bones. We then constructed two 3D surfaces by meshing curves on successive slices. Finally, we regularized the meshed surfaces using YAMS.

3.1. Image segmentation. To locate the tissue of interest (bone or soft tissue) on scan slices, we use a threshold technique. Given the type of radiological images and their acquisition, this step is relatively simple. The X-ray data acquisition protocol guarantees that grayscale values of tissue of interest belong to well-differentiated sections. These grayscale values are determined in Hounsfield Units (HU) and are referenced in the literature. They remain stable between different acquisitions [20]. According to this scale, fat is around $-100 \mathrm{HU}$, muscle around $+100 \mathrm{HU}$, trabecular bone ranges from +100 to $+300 \mathrm{HU}$ and cortical bone reaches around $+2000 \mathrm{HU}$.

To segment images, they are thresholded using Hounsfield Unit limits. For this operation, a pixel is considered to correspond to bone if the grayscale value is greater than $350 \mathrm{HU}$ and to soft tissue if the grayscale value is less than -200 HU. Pixels with values between -200 and $350 \mathrm{HU}$ are considered to be outside tissues of interest, in a part referred to as image background. Slice thresholding makes it possible to create two binary images: one separating the background from bone, and the other separating background from soft tissue. By convention, the pixels of these binary images situated on the background have a value of 1 , and those on an area of interest have a value of 0 (the interest of this convention will appear next in Equation 1). Segmentation is illustrated in Figure 2.

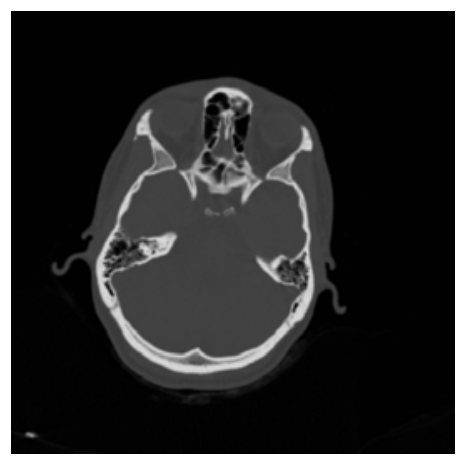

(a)

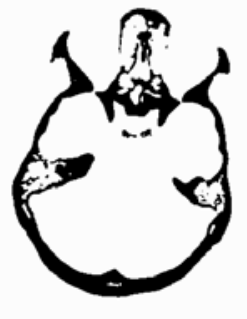

(b)

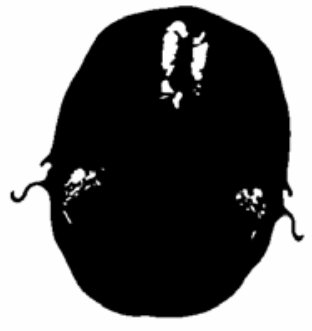

(c)

Figure 2. Segmentation of a CT-scan. (a) slice, segmentations of (b) the bone tissues and (c) the soft tissues.

Any defects are removed from images after segmentation. X-ray artefacts caused by dental fillings (see Figure 3) are removed manually from each slice. Small areas outside the head caused by dust are removed automatically by finding the largest 

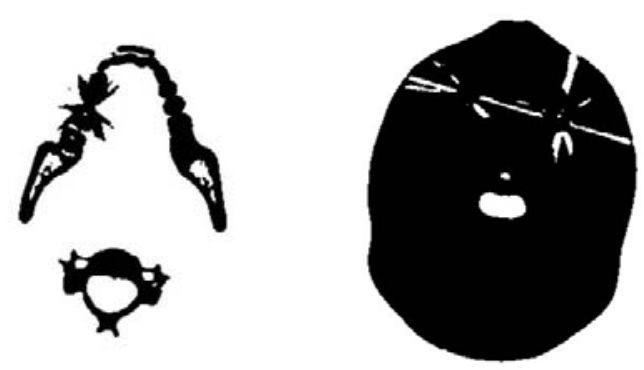

FiguRE 3. X-ray artefacts caused by metallic dental implant fixtures.

connecting area formed by soft tissue pixels and by locating the pixels of interest outside that area.

3.2. Contour extraction. After segmentation, we extract separately two curves from binary images: one wrapping bone and another wrapping soft tissue. The two curves are constructed using the same procedure and complying with several constraints:

- construction of closed curves that wrap the areas of interest and fill the holes and hollows they contained,

- curves have to fit snugly along the borders of the areas of interest (bone or soft tissue).

Wrapping is obtained by evolving a curve to completely cover the area of interest. This is done using three forces. As seen in Figure 4, the first of these forces draws those curve points which are not set on the area of interest towards the inside of the curve. This extraction force is constant at all the points it acts upon and is always oriented in the direction of the internal normal of the curve at a given point. Since the area of interest is always inside the curve, the extraction force pushes the curve towards the borders of the area where it will settle.

The second force, referred to as the adhesion force, adds to the extraction force on those curve points which have a neighbouring point already located on a border of interest. It thus reinforces the adhesion of the curve to the areas of interest. Like the extraction force, it acts in the direction of the internal normal of the curve and is constant.

Extraction and adhesion forces constitute what we refer to as data driven forces enabling the curve to be guided accurately towards the borders of the area to be wrapped.

These two forces alone are not sufficient to extract the curve. When applied alone, they tend to drag the curves towards the inside of the holes and hollows in the areas of interest. This is why a third force is needed to attenuate this phenomenon.

To define this force, we propose the following: when a curve evolves in a hollow under the effect of extraction and adhesion forces, its curvature at each point tends to 

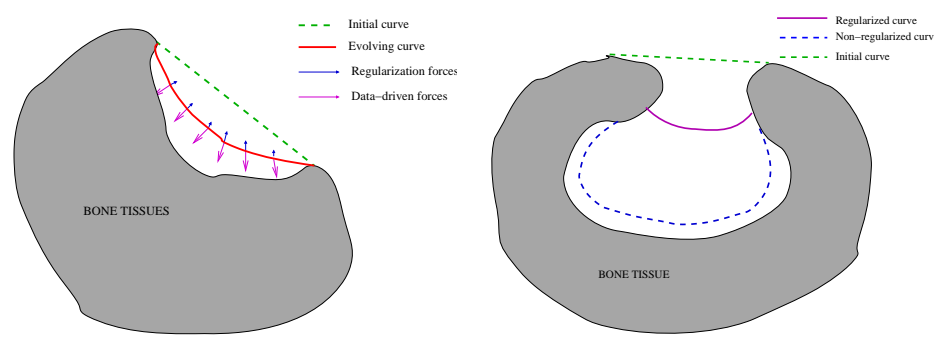

Figure 4. Dynamics of curve evolution.

increase making it more and more irregular. Consequently, by regularizing the curve, it is possible to counterbalance the effect of these two forces inside the hollow. Hence, the third force we applied was a curve regularization force, or more specifically, a mean curvature regularization force: it is applied at each point of the curve not already fixed on an area of interest, in the direction of the normal and in the direction given by the sign of curvature, and proportional to the cube root of the curvature.

This force tends to reduce curvature all along the curve. When the regularization force acts alone, the curve evolves towards a segment on the right (curve with zero curvature at all points). When the regularization force is applied with the extraction and adhesion forces, the curve evolves following a compromise between regularity and adjustment of precision at the borders of the area of interest.

Curve evolution is written mathematically as an ordinary differential equation:

$$
\frac{d C}{d t}(t)=E(C(t))\left(p_{1} k^{\frac{1}{3}}(t)+p_{2}+p_{3}\left(\frac{d E}{d s^{+}}(C(t))+\frac{d E}{d s^{-}}(C(t))\right)\right) N(t) .
$$

In the right part of the equation, $C(t)$ represents the curve at time $t$, and $\frac{d C}{d t}(t)$ its temporal derivative, which represents its evolution over time. The left part of the equation describes the forces acting on the curve. The term $E$ is one of the binary images obtained after segmentation. The term $E(C(t))$ weights the forces acting on point $\mathrm{C}(\mathrm{t})$ of the curve. As this term equals 0 if $C(t)$ is on an area of interest (bone or soft tissue), it cancels the forces when a curve point reaches an area of interest. Thus the curve can be fixed on a given area of interest. The quantity $N(t)$ is the internal normal of the curve at the point $C(t)$. It gives the direction of curve evolution at each point. In this direction, the evolution speed depends mainly on other terms, indicating the forces present:

- $k^{\frac{1}{3}}(t)$ is the regularization force, $k(t)$ being the signed curvature of the curve, and $p_{1}$ is a positive constant weighting regularization.

- the second term, which is constant, is the extraction force. It is weighted by $p_{2}$.

- $\left(\frac{d E}{d s^{+}}(C(t))+\frac{d E}{d s^{-}}(C(t))\right)$ is the adhesion force. The values $\frac{d E}{d s^{+}}(C(t))$ and $\frac{d E}{d s^{+}}(C(t))$ represent derivatives of the image $E$ on the right and left of the curve point and along the curve. These derivatives are different to zero when the neighbouring points on the right or left of a curve point have a different 


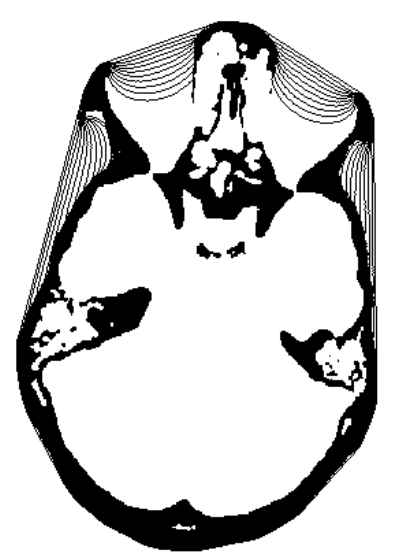

FIGURE 5. Contour extraction by curve evolution.

label to that of the point. If this is not the case, there is no adhesion force. If this is the case, the term produces an adhesion force that adds to the extraction force. $p_{3}$ is a positive constant that determines the weight of the adhesion force.

To apply curve evolution to the computation of bone and soft tissue wrap, we proceed as follows: we first calculated the convex hull of the area of interest (blue curve in Figure 5, i.e. the polygon that covers the area of interest and whose vertices are all located on the external border of the area. This constitutes effective initialization for computation of the wrap.

Then, using the equation given above, we let the curves evolve between the successive vertices of the convex hull. We use as many evolution curves as there were pairs of successive vertices on the convex wrap. Curves are generated between each pair of vertices using the segment linking the two vertices. The curves then evolve under the effect of evolution forces but remain linked to the two vertices by their extremities.

We evolve all the curves until they stabilized with a high regularization weight $\left(p_{1}\right)$ that forced the curves to remain smooth. After evolution of all the curves, we observed the results obtained. We fixed a curve if:

Condition $\left(C_{1}\right)$ : it accurately followed the external border of an area of interest.

Condition $\left(C_{2}\right)$ : it did not intersect another curve.

If the curve does not comply with condition $\left(C_{2}\right)$, we cancel the result of curve evolution and replace the curve obtained by the previous curve, which is then fixed. If condition $\left(C_{2}\right)$ complies, but not condition $\left(C_{1}\right)$, we let the curve evolve again with a lower regularization weight, in order to bring the curve closer to the area of interest. 
After inspecting all the curves, we then launch new evolution equations for all non-fixed curves. When these evolutions are completed, we again check conditions $\left(C_{1}\right)$ and $\left(C_{2}\right)$ and determine new curves to fix. We repeat this procedure several times, each time reducing the regularization weight value until it reach the lower limit or until no further curve can evolve.

The different areas of the craniofacial block or of the face do not all have the same curvature characteristics. So it is difficult to select one regularization weight value to calculate wraps for different slices. By using the repetitive procedure described above, it is possible to overcome this problem: by gradually increasing the value of the extraction force $p_{2}$, we adjusted regularization weight locally on each area of the craniofacial block or of the face and are able to control the maximum curvature authorized on the curves under investigation. This value can be set as a function of the areas where surface curvature (bone or soft tissue) is the greatest.

Furthermore, condition $\left(C_{2}\right)$ of the procedure described above makes it possible to avoid the curve intersecting that occurs when bony areas present holes. These intersections generate irregularities in the wrap that reduce the quality of the extracted surfaces.

In practice, the extracted curve presents as a closed polygon (ordered series of points). The space between the points is less than a pixel, thus guaranteeing subpixel accuracy. We choose to calibrate equation parameters empirically: regularization weight value $p_{1}$ is equal to the maximum curvature reached by the polygon. In this way, the regularization term is always below 1 in absolute value. The adhesion force weight value $p_{3}$ is set at 0.7 for bone and at 0.95 for soft tissue. The extraction force weight value $p_{2}$ is allowed to range between 0.05 and 0.15 for bone, and 0.1 and 0.3 for skin.

3.3. Surface construction. After the above step, we obtain a closed polygon (ordered series of points) for each slice. This polygon is a discrete approximation of the external contour of the object to be located on the slice. The problem then remains of constructing a preliminary meshing for the underlying surface, using two successive discrete contours. It should be noted at this stage that the underlying surface is not available since the acquisition method on a scanner only generates slices. It is therefore impossible to obtain a surface construction corresponding to biological situations. Having said that, several surface constructions between two successive curves can be envisaged. Each construction has advantages and disadvantages. A good construction should favour biological reality even if it slower.

The method we developed is based on the following biological hypothesis: the surfaces formed by skin or bones are smooth and vary only slightly locally, i.e. curvature is low. This hypothesis corresponds to the mathematical notion of minimal surface. The standard representation of a minimal surface consists in imagining the part of a soap bubble resting between a set of fixed points. In our case, the fixed points are those points between two successive contours and we look for an approximation of the minimal surface resting on these points. As seen in Figure 6, this 


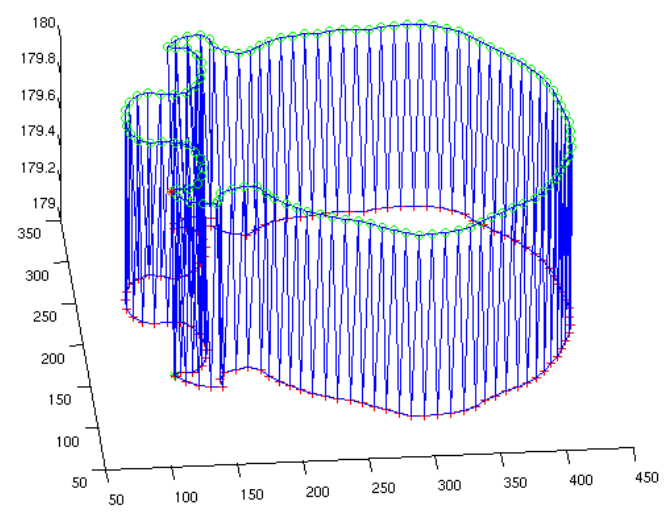

Figure 6. Construction of the surface mesh.

approximation is obtained in the form of a triangulated mesh (made up of small triangles; the vertex of one of the triangles resting on one of the two curves and the vertices of the other two triangles on two consecutive points of the other curve). Given the two successive contours (discretized), the mesh approximating the minimal surface between these two contours is obtained using a dynamic programming algorithm. This algorithm is described in appendice B

A first complete mesh is constructed by stacking these minimal meshes between the contours of successive slices. It is important to note that (1) contours are constructed from series of points less than a pixel apart, (2) two successive contours are separated from the pitch scanner. Thus, the distance of the constructed mesh from the real surface (non observed) are 0.8 millimeter.

The mesh obtained by stacking meshes approximating minimal surfaces has the disadvantage of being over-adjusted to the underlying geometry of the image, i.e. to the pixels. It presents the classical artefacts observed with this type of construction (staircase artefacts). To fix this drawback, regularization has to be applied. This step is performed using YAMS software designed by Pascal Frey, which regularizes mesh (curvature, size and geometry of triangles) whilst preserving good geometric qualities $[13,12]$. This software is known to provide small deformation around 2 time the local mean value of the original face lengths. The following commands were used:

(1) yams -O 9 maillage.mesh -nr. This command denoises the mesh (removed the effect of pixelization).

(2) yams -O 1 -e -f maillage.mesh. This command regularizes mesh by controlling its density by curvature. This regularization constructs approximately equilateral triangles which are easy to use for digital computations.

(3) yams -O 2 -e -f maillage.mesh. This command regularizes mesh by preserving a Hausdorff distance criterion between sets. The meshes generated by this 
regularization are much lighter than the previous mesh in terms of numbers of vertices.

In practice, step (1) is followed by one of the regularization commands (2 or 3 ).

Following surface extraction, anatomical landmarks are transformed in the mesh by taking account of pixel size, slice thickness and any deviation to the initial slice. This transformation is obtained by projecting the anatomical landmark onto the mesh. The mesh is modified so that the projected anatomical landmark become a vertex of the mesh. Here, the thinness of the meshes constructed earlier is of prime importance. The technique developed ensured a deviation between anatomical landmarks and their correspondence with the mesh of 0.8 millimeter.

3.4. Tissue thickness. For each sum of bone surface mesh, the external normals and their intersection with skin surface mesh are calculated. At each landmark of the bone surface, soft tissue thickness are defined along the external normal. We then have an individual thickness value with real positive values defined on the bone surface function.

3.5. Surface geodesics and patchs. With our system, it is also possible to calculate geodesics on the meshed surface and extract anatomically identified parts from the bone surface (bone patch).

In geometry, geodesics refers to the shortest path between two points of a surface. The usual example given is that of the meridians which are the geodesics between the North and South poles. Determining the geodesics on an area defined by a mesh is a complex task. There are several algorithms for calculating mesh geodesics.

Because of the problem of digital stability, we had to combine two methods to calculate the geodesics on our mesh: the Surazhsky algorithm [39] and the Fast Marching Algorithm [37] implemented by Peyré in the Geowave library. Using a beam-following technique, the first algorithm obtained accurate geodesics between two points. However, the authors' implementation is not available, so we wrote a Matlab code which in practice was rather slow, so impractical for computation of a large number of geodesics with points relatively spaced out on the mesh. The second algorithm is very rapid and provides good visual approximation of geodesics. However, the curves obtained are quite irregular locally when close to the two points concerned, thus making it difficult to extract the surface later. To correct this defect, we use the first algorithm to refine the geodesics line obtained by the second algorithm near the source point and the target point.

Computation of geodesics facilitates bone-patch extraction. We use the anatomical landmarks on the mesh together with the geodesics successively linking these landmarks. Bone-patches are extracted from the mesh by simply locating the closed surfaces bordered by the geodesics between the anatomical landmarks. 


\section{Results}

We applied the surface extraction techniques described in Section 3 to all the CTscans in our database. Some meshes of skull and face surfaces are shown in Figure 7. As illustrated in Figure 8, we also extracted some predefined anatomical patches on skull surfaces. We also measured soft tissue depths at each anatomical landmark of the extracted skull surfaces, using the technique described in Section 3.4. This step is illustrated in Figure 9. Statistics of these measured thicknesses are reported in Table 4. In Table 5, our measurements are compared to those of several other studies.

We found that soft tissue thickness presented low variability at the following landmarks: Glabella, Nasion, Nasale, Euryon, Infradentale, Point B, Ectoconchion et Orbitale Superius.

The greatest variability in soft tissue thickness was observed at the following landmarks: Nasospinale, Anterior Nasal Spine, Prosthion, Incisor Superius, Gonion, Ectomolare, Alare, Infraorbitale, Condylion, Orbitale and Jugale. This high variability can be explained by several factors. Certain facial area, particularly the cheeks, may have large amounts of adipose tissue if subjects gain weight. The variation in soft tissue thickness at landmarks in these areas is therefore correlated to subjects'bodyweight. The landmarks concerned are: Infraorbitale, Ectomolare, Condylion, Gonion and Jugale. Marked variability in soft tissue thickness is also observed for points on, or close to, dental crowns. These thickness values depend on the position of teeth, which vary between subjects. This is seen with the Incisor Superius which is the most salient point on the crown of central maxillary incisors in the sagittal median plane. The position of the maxillary incisors varies as a function of lingual and labial muscle activity, any orthodontic treatment or oral parafunctions. Furthermore, soft tissue thickness at Incisor Superius and Prosthion points depends on the position of lips and labial muscle contraction during the tomodensitometric examination.

Since Point A, Nasospinale and the Anterior Nasal Spine point are situated on bone sites where the curvature is very marked, we observed greater variability in the normal and consequently a projection error of this normal on the skin surface. Projection errors of the normal were also seen on the Gonion landmark due to the morphology of the mandibular angle. The deep layer of the superficial masseter muscle superficiel is attached at the mandibular angle by strong tendinous cones [14]. These cones leave a bone print in the form of vertical crests. Hence, muscle power has an impact on the morphology of the mandibular angle by sometimes laterally deforming the bone rim on which the Gonion landmark is situated. This added to the fact that it was difficult to calculate a reliable normal at this landmark because of bone curvature. 

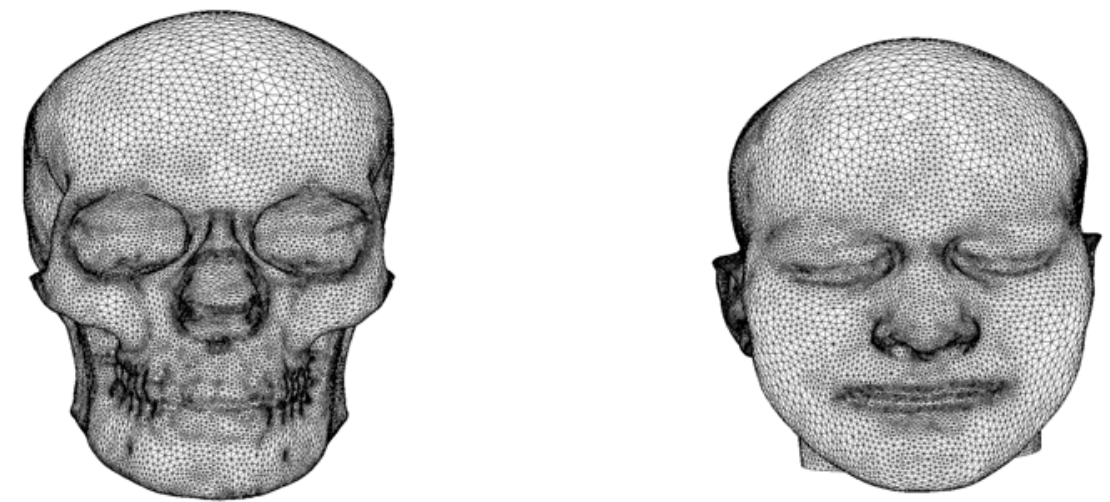

FiguRE 7. Examples of skull and face surfaces extracted from CT-scans.
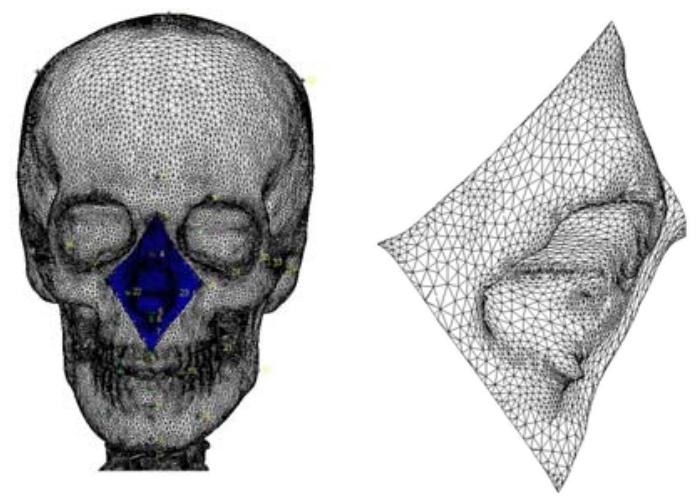

FIGURE 8. Example of the nose patch on the bone surface.

\section{DisCUSSION}

5.1. Surface extraction. Three-dimensional image segmentation is one of the major problems in Image Processing. Since the 90's, a wide variety of techniques has been proposed, ranging from variational techniques, such as snakes [6] or level-sets and fast-marching [36], to statistical techniques such as those based on Markov random fields [48]. All techniques have been successfully applied to the challenging problem of segmentation of head structures in MR images (white and gray matter of the brain, bones, etc.) $[24,25,36,47,48]$. Some powerful platforms have also been developed for these specific applications: BRAINVISA [7] and BT2 [21].

In CT-scans, the skull is a well-defined structure. As described in Section 3.1, bone tissue can easily be separated from soft tissue using Hounsfield Units. For this 

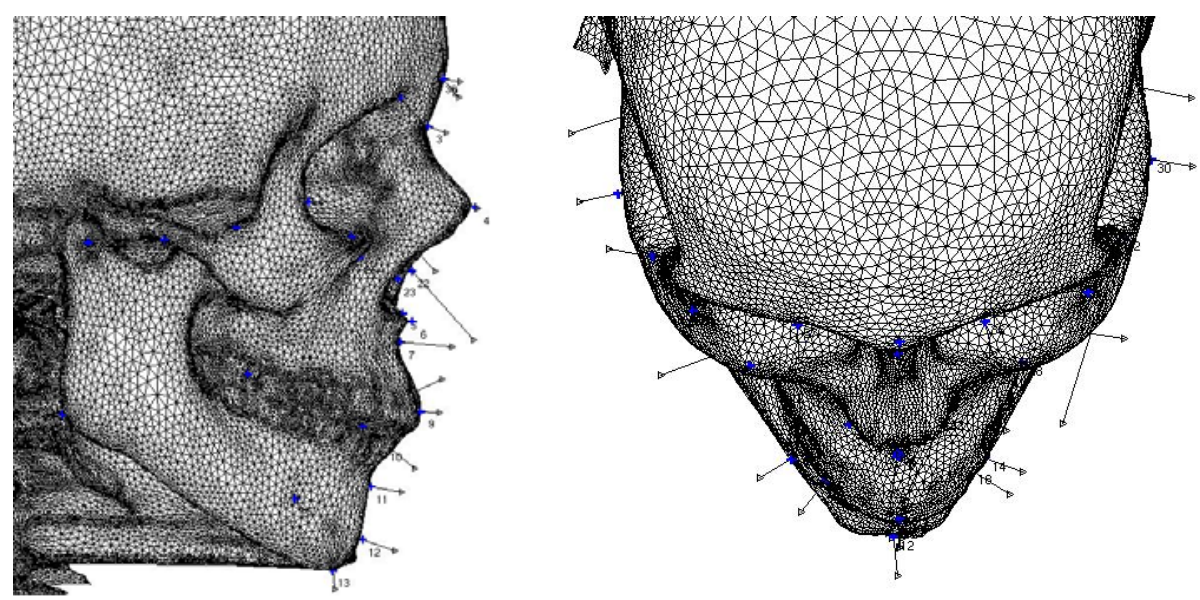

FIGURE 9. A skull mesh with anatomical landmarks and associated soft tissue thicknesses in normal directions.

reason, the segmentation of the skull from CT images is far less difficult than the segmentation of brain structures from MRI. Medical visualization software (e.g. Osirix [33]) frequently includes efficient skull segmentation and meshing tools. Nevertheless, surfaces obtained with such tools cannot be easily used for facial reconstruction for two main reasons. First, surfaces cover the whole bone borders without making any distinction between the inner and outer borders. Second, they have as many holes as do skulls. Hence the real difficulty we are faced with is the extraction of a closed and smooth surface which wraps the external skull border without any hole.

In order to obtain skull surfaces having such properties, Vandermeulen et al. used morphological operations [43]. In such an approach, the choice of the structuring element is critical. If it is too small, skull holes may not be filled and artefacts cannot be removed. If it is too large, morphological filtering might change the regular bone structure morphology. Compared to this work, our technique has several advantages: (1) it guarantees that the constructed skull surface covers all the holes on the skull, whatever their size, (2) due to terms in the equation used for contour extractions, the extracted surface fits accurately to the real external skull surfaces at places where there is no hole, (3) the extracted surface is represented by a mesh, which is smoothed so as to alleviate discretization artefacts.

5.2. Thickness measurements. The first research into the evaluation of soft tissue thickness was performed by the German anatomist Welcker in 1883 [46] who measured nine points on the median sagittal line of 13 male cadavers using a knife blade. His continued this work by further assessing the layers of soft tissue at lateral points using a needle (sample size: 28 cadavers, 24 men and four women) [19]. His was the first author to introduce the notion of body size by recording the same measurements in a second sample of 24 men of medium size and nine thin men. The work carried out by His was continued by Kollman and Buchly [23] who added 


\begin{tabular}{|c|c|c|c|c|c|}
\hline & & Mean & Min & Max & Std \\
\hline 1 & Vertex & 5.01 & 2.93 & 8.45 & 1.51 \\
2 & Glabella & 3.72 & 2.65 & 4.76 & 0.54 \\
3 & Nasion & 5.56 & 3.79 & 8.30 & 0.94 \\
4 & Nasale / Rhinion & 1.25 & 0.06 & 4.13 & 0.90 \\
5 & Nasospinale & 11.18 & 7.21 & 19.13 & 2.39 \\
6 & Anterior nasal spine & 9.55 & 6.40 & 17.51 & 2.72 \\
7 & Point A & 11.30 & 8.08 & 15.88 & 1.78 \\
8 & Prosthion & 10.71 & 6.08 & 16.43 & 2.40 \\
9 & Incisor superius & 5.91 & 0.95 & 10.67 & 2.38 \\
10 & Infradentale & 10.49 & 7.22 & 13.78 & 1.37 \\
11 & Point B & 9.34 & 7.07 & 13.15 & 1.31 \\
12 & Pogonion & 8.65 & 4.78 & 11.61 & 1.58 \\
13 & Gnathion & 5.20 & 2.17 & 9.63 & 1.57 \\
14 & Left mentale & 10.36 & 7.65 & 13.78 & 1.56 \\
15 & Right mentale & 9.82 & 6.63 & 13.31 & 1.63 \\
16 & Left gonion & 14.80 & 7.61 & 24.27 & 3.60 \\
17 & Right gonion & 13.79 & 6.27 & 31.65 & 4.85 \\
18 & Left canine & 8.55 & 3.94 & 12.39 & 2.08 \\
19 & Right canine & 7.64 & 2.25 & 13.23 & 2.34 \\
20 & Left ectomolare & 22.13 & 12.99 & 28.90 & 2.84 \\
21 & Right ectomolare & 21.48 & 12.98 & 28.41 & 3.01 \\
22 & Left alare & 11.25 & 5.60 & 20.87 & 4.86 \\
23 & Right alare & 9.76 & 5.02 & 19.78 & 4.44 \\
24 & Left infraorbitale & 12.88 & 7.03 & 20.12 & 2.96 \\
25 & Right infraorbitale & 12.94 & 6.69 & 18.93 & 2.96 \\
26 & Left lateral condylion & 13.68 & 8.40 & 21.44 & 3.16 \\
27 & Right lateral condylion & 12.87 & 7.34 & 18.80 & 2.85 \\
28 & Left orbitale & 5.61 & 2.46 & 19.23 & 3.34 \\
29 & Right orbitale & 4.86 & 1.91 & 14.95 & 2.62 \\
30 & Left zygion & 8.12 & 4.77 & 13.87 & 1.86 \\
31 & Right zygion & 7.11 & 3.55 & 13.21 & 1.89 \\
32 & Left jugale & 10.83 & 6.34 & 19.80 & 2.96 \\
33 & Right jugale & 9.61 & 5.23 & 16.22 & 2.47 \\
34 & Left ectoconchion & 9.34 & 7.07 & 13.15 & 1.31 \\
35 & Right ectoconchion & 4.05 & 0.83 & 8.47 & 1.65 \\
36 & Left orbitale superius & 6.65 & 4.20 & 12.23 & 1.52 \\
37 & Right orbitale superius & 6.63 & 4.43 & 9.74 & 1.22 \\
38 & Left euryon & 4.03 & 2.15 & 7.31 & 1.10 \\
39 & Right euryon & 3.09 & 1.48 & 5.15 & 0.92 \\
\hline
\end{tabular}

TABLE 4. Soft tissue thickness measurements $(\mathrm{mm})$ on anatomical landmarks from 47 women aged between 20 and 40 years.

supplementary landmarks and noted their measurements more accurately for eight women and 45 men using a needle charred by a candle flame. They interpreted their results by a statistical study taking account of variations in soft tissue thickness as a function of body size. 


\begin{tabular}{|c|c|c|c|c|c|c|c|}
\hline \multicolumn{2}{|r|}{ Reference studies } & 1 & 2 & 3 & 4 & 5 & 6 \\
\hline \multicolumn{2}{|r|}{ Number of cases } & $(\mathrm{N}=47)$ & $(\mathrm{N}=25)$ & $(\mathrm{N}=25)$ & $(\mathrm{N}=234)$ & $(\mathrm{N}=52)$ & $(\mathrm{N}=15)$ \\
\hline 1 & Vertex & 5.01 & & & & & \\
\hline 2 & Glabella & 3.72 & 5.60 & 5.03 & 5.06 & 4.80 & 4.70 \\
\hline 3 & Nasion & 5.56 & 6.69 & 5.66 & 6.2 & 5.50 & 5.30 \\
\hline 4 & Nasale / Rhinion & 1.25 & 2.40 & 2.92 & 2.64 & 1.80 & 1.60 \\
\hline 5 & Nasospinale & 11.18 & & & & & \\
\hline 6 & Anterior nasal spine & 9.55 & & & & & \\
\hline 7 & Point A & 11.30 & 13.28 & 8.14 & 9.64 & 9.10 & 7.40 \\
\hline 8 & Prosthion & 10.71 & 11.23 & 8.92 & 9.79 & & \\
\hline 9 & Incisor superius & 5.91 & & & & & \\
\hline 10 & Infradentale & 10.49 & 12.00 & 10.10 & 10.89 & & \\
\hline 11 & Point B & 9.34 & 10.61 & 9.99 & 9.69 & 10.30 & 9.60 \\
\hline 12 & Pogonion & 8.65 & 10.19 & 10.34 & 9.76 & 9.20 & 9.20 \\
\hline 13 & Gnathion & 5.20 & 7.15 & 5.94 & 5.92 & 6.00 & 5.40 \\
\hline 14 & Left mentale & 10.36 & & & & & \\
\hline 15 & Right mentale & 9.82 & & & & & \\
\hline 16 & Left gonion & 14.80 & 9.10 & 11.82 & 14.67 & 17.40 & 15.30 \\
\hline 17 & Right gonion & 13.79 & & & & & \\
\hline 18 & Left canine & 8.55 & & & & & \\
\hline 19 & Right canine & 7.64 & & & & & \\
\hline 20 & Left ectomolare & 22.13 & & & & & \\
\hline 21 & Right ectomolare & 21.48 & & & & & \\
\hline 22 & Left alare & 11.25 & & & & & \\
\hline 23 & Right alare & 9.76 & & & & & \\
\hline 24 & Left infraorbitale & 12.88 & & & & & \\
\hline 25 & Right infraorbitale & 12.94 & & & & & \\
\hline 26 & Left lateral condylion & 13.68 & & & & & \\
\hline 27 & Right lateral condylion & 12.87 & & & & & \\
\hline 28 & Left orbitale & 5.61 & & & & & \\
\hline 29 & Right orbitale & 4.86 & & & & & \\
\hline 30 & Left zygion & 8.12 & 5.01 & 7.68 & 6.98 & 7.40 & 4.90 \\
\hline 31 & Right zygion & 7.11 & & & & & \\
\hline 32 & Left jugale & 10.83 & & & & & \\
\hline 33 & Right jugale & 9.61 & & & & & \\
\hline 34 & Left ectoconchion & 9.34 & & & & & \\
\hline 35 & Right ectoconchion & 4.05 & & & & & \\
\hline 36 & Left orbitale superius & 6.65 & & & & & \\
\hline 37 & Right orbitale superius & 6.63 & & & & & \\
\hline 38 & Left euryon & 4.03 & & & & & \\
\hline 39 & Right euryon & 3.09 & & & & & \\
\hline
\end{tabular}

TABLE 5. Comparison of our soft tissue thickness measurements with those of other studies. 1: Tilotta et al, 2: Helmer et al., 3: Rhine and Moore, 4: De Greef et al., 5: Manhein et al. (women aged between 19 and 34 years), 6: Manhein et al. (women aged between 35 and 45 years). 
Important information on soft tissue evaluation was provided by the studies performed by the anthropologist, archeologist and sculptor Gerasimov [15, 16]. He recorded thickness values for large samples of Caucasian-type or Mongol-type cadavers, differentiated according to age, for which death had occurred within the previous 24 hours. In this way, Gerasimov evidenced a low variation in soft tissue thickness as a function of age.

More recently, Rhine and Campbell constituted a population of 59 Black Americans, deceased within the previous 12 hours and recorded measurements at ten landmarks on the profile and 22 lateral landmarks [35].

These old methods for evaluating soft tissue thickness are of great interest for facial reconstruction. The disadvantage, however, lies in the fact that they were taken from cadavers. In the hours following death, tissues dehydrate and droop due to loss of muscle activity and zero gravity. This results in any measurements taken after death being slightly less than real values. Also, the rigidity caused by irreversible biochemical transformations gradually affects all striated and smooth muscle and usually starts within a mean of three hours of death. It is complete within 8 - 12 hours and remains for 12 - 36 hours, then gradually fades. These timelines and the intensity of the rigor mortis varies depending on the age of the subject, the cause of death and the ambient temperature [10].

The lack of precision of values recorded from cadavers is seen from the discrepancy between our results and those obtained by Rhine and Moore in [34] who conducted a study on 25 women and taking body size into account.

Phillips and Smuts recorded soft tissue measurements using scans from a mixedblood population in South Africa [31]. Although their results are not comparable to ours because of the different origins of the population, they also showed the interest of using this method on live subjects in comparison with measurements on cadavers.

De Greef et al. conducted a study using ultrasound on a sample of 234 women aged 18 to 29 years and 72 women aged 30 to 39 years. They measured mean soft tissue thickness at ten landmarks on the profile and 21 lateral landmarks [8]. Ultrasound not being an invasive technique, the principal advantage is that numerous data can be collected and criteria such as age, sex or body weight statistically integrated [4]. However, the major disadvantage of the De Greef's technique is the great difficulty of reliably fixing the bone reference landmarks since measurements are taken from skin landmarks. From this view point, our approach is radically different : the soft tissue depths are computed taking into account not only anatomical points referenced on the skull surface but also the geometry of the skull surface described by normals of the triangulated surfaces. In their study, nine landmarks on the profile and two lateral landmarks were similar to those in our study. Our results were comparable to those of De Greef, except the values observed for Point A, Infradentale and Zygion, which were landmarks we considered to be unstable because of the difficulty involved in calculating a reliable normal or the high correlation with body size or teeth position. 
Manhein also determined mean soft tissue thickness using an ultrasound technique on a population of 52 women aged 19 to 34 years and 15 women aged 35 to 45 years [26]. The same discrepancy was observed between values obtained by this author in values for Point $\mathrm{A}$ and Zygion and our values. We also obtained a Nasion value that was greater than the value recorded by Manhein, whereas it is similar to that obtained in $[8,34]$. The other points were similar.

Helmer evaluated soft tissue thickness using a scanner in a population of twelve women aged 20 - 29 years and 13 women aged 30 - 39 years [18]. Nine odd-numbered landmarks and two even-numbered landmarks were the same as in our study. Our values were slightly lower than those of Helmer, except for the Zygion.

As mentioned in introduction, soft tissue depth measurements provide a data which is helpful for facial reconstruction techniques [3, 11, 38, 44, 45]. However, such measurements are always sparsely distributed over the skull surface and usually located in some specific parts of the skull where anatomical points can be reliably located. Hence, information brought by measurements is not sufficient for having an accurate facial reconstruction on the whole skull surface. The triangulated surfaces we extract from CT-scans offer a more complete information which can lead to better reconstructions in between anatomical points.

\section{Conclusion}

We described a new public database which is currently composed of 85 CT-scans of healthy subject aged 20 to 65 years old. We also presented some image-processing techniques which allowed us to extracted some triangulated surfaces of skull and face of each subject. These extracted surfaces were also included in the database. Using them, we computed soft-tissue depths at known landmark positions. We compared our measurements to those of Rhine et al., Helmer et al. De Greef et al. and Manhein et al., pointing out similarities and deviations.

The construction of the complete database (acquisition of CT-scans, extraction of surfaces, soft-tissue depth measurements) is the keystone of a project conducted by our multidisciplinary team [40]. Next step of our project will concern the construction of statistical techniques for computer-based facial reconstruction, taking full advantage of the constructed database.

\section{REFERENCES}

[1] M. Berar, M. Devisgnes, G. Bailly, and Y. Payan. 3D semi-landmarks-based statistical face reconstruction. Journal of Computing and Information Technology, 1:31-43, 2006.

[2] T. Buzug. Special issue on computer assisted craniofacial reconstruction and modelling : Editorial. Journal of Computing and Information Technology, 14(1):1-7, Mars 2006.

[3] P. Claes, D. Vandermeulen, S. De Greef, et al. Craniofacial reconstruction using a combined statistical model of face shape and soft tissue depths: methodology and validation. Forensic Science International, 159S:147-148, 2006. 
[4] P. Claes, D. Vandermeulen, S. De Greef, et al. Craniofacial reconstruction using a combined statistical model of face shape and soft tissue depths: methodology and validation. Forensic Science International, 159S(1):S147-S158, 2006.

[5] J.G. Clement and M.K. Marks, editors. Computer-Graphic CranioFacial Reconstruction. Academic Press, 2005.

[6] L. Cohen. On active contour models and balloons. Computer Vision, Graphics, and Image Processing: Image Understanding, 53(2):211-218, 1991.

[7] Y. Cointepas, J.-F. Mangin, L. Garnero, et al. BrainVISA: Software platform for visualization and analysis of multi-modality brain data. NeuroImage, 13(6):98, 2001.

[8] S. De Greef, P. Claes, D. Vandermeulen, et al. Large-scale in-vivo caucasian facial soft tissue thickness database for craniofacial reconstruction. Forensic Science International, 159S:S126S146, 2006.

[9] S. De Greef and G Willems. Three-dimensional cranio-facial reconstruction in forensic identification: Latest progress and new tendencies in the 21st century. Journal of Forensic Sciences, 50(1):1-6, 2005.

[10] M. Durigon. Pratique médico-légale. Masson, Paris, 1999.

[11] R. Evenhouse, M. Rasmussen, and M Sadler. Computer-aided forensic facial reconstruction. Journal of BioCommunication, 19(2):22-28, 1992.

[12] P.J. Frey, About surface remeshing, In Proc. of the 9th Int. Meshing Roundtable, New Orleans, LO, USA, 123-136, 2002.

[13] P.J. Frey Yams : A fully Automatic Adaptive Isotropic Surface Remeshing Procedure, Technical Report 0252, INRIA Rocquencourt, novembre, 2001.

[14] J-F. Gaudy. Anatomie clinique. CdP, Rueil-Malmaison, 2003.

[15] M.M. Gerasimov. Vosstanavleni litsa po tcheripou. Izdituekstvi Akadmii Naouk Sssr, Moscou, 1955.

[16] M.M. Gerasimov. The face finder. J.B. Lippincott, 1971.

[17] W.D. Haglund and D.T. Reay. Use of facial approximation techniques in identification of green river serial murder victims. American Journal of Forensic Medicine and Pathology, 12(2):132$142,1991$.

[18] R. Helmer. Schödelidentifizierung durch elektronische bildmischung. Kriminalistik Verlag GmbH, Heidelberg, 1984.

[19] W. His. Anatomische forschungen ueber johann sebastian bachos gebeine und antliz nebst bemerkungen ueber dessen bilder. abhandlungen der mathematisch-physikalischen klasse der konigl. Sachsischen Gesellschaft der Wissenchaften, 22:379-420, 1895.

[20] G.N. Hounsfield. Computerized transverse axial scanning (tomography): Part i. description of system. Br. J. Radiol., 46:1016-22, 1973.

[21] M. Jenkinson, M. Pechaud, and S. Smith. BET2: MR-based estimation of brain, skull and scalp surfaces. In Eleventh Annual Meeting of the Organization for Human Brain Mapping, 2005 .

[22] M.W. Jones. Facial reconstruction using volumetric data. In Proc. of the 6th International Vision Modelling and visualisation Conference, pages 136-142, Stuttgart, 2001.

[23] J. Kollmann and W. Buchly. Die persistenz der rassen und die reconstruction der physiognomie prahistorischer schadel. Archiv für Anthropologie, 25:329-359, 1898.

[24] R. Malladi, R. Kimmel, D. Adalsteinsson, et al. Geometric approach to segmentation and analysis of 3D medical images. In Proc. of the Workshop on Mathematical Methods in Biomedical Image Analysis (MMBIA'96), pages 244-252, San Francisco, CA, USA, 1996.

[25] R. Malladi and J. Sethian. A real-time algorithm for medical shape recovery. In Proc. of the International Conference on Computer Vision (ICCV'98), pages 304-310, 1998. 
[26] M.H. Manhein, G.A. Listi, R.E. Barsley, et al. In vivo facial tissue depth measurements for children and adults. Journal of Forensic Sciences, 45(1):48-60, 2000.

[27] R. Martin and K. Saller. Lehrbuch der Anthropologie in Systematischer Darstellung. Stuttgart. Gustav Fisher Verlag, 1956.

[28] C. Menin. La population gallo-romaine de la nécropole de Maule (France, Yvelines) : étude anthropologique. PhD thesis, University Paris 6, Pierre et Marie Curie, France, 1977.

[29] L.A. Nelson and S.D. Michael. The application of volume deformation to three dimensional facial reconstruction: a comparison with previous technique. Forensic Science International, 94:167-181, 1998.

[30] E. Peyre. Etude anthropologique qualitative et quantitative de la population mérovingienne de la nécropole de Maule (France, Yvelines). PhD thesis, University Paris 6, Pierre et Marie Curie, 1977.

[31] V.M. Phillips and N.A. Smuts. Facial reconstruction : utilization of computerized tomography to measure facial tissue thickness in a mixed racial population. Forensic Science International, 83:51-59, 1996.

[32] G. Quatrehomme, S. Cotin, G. Subsol, et al. A fully three-dimensionnal method for facial reconstruction based on deformable models. Journal of Forensic Sciences, 42:649-625, 1997.

[33] O. Ratib, A. Rosset, and J. Heuberger. OSIRIX: the pocket guide. 2007.

[34] J. Rhine and C.E. Moore. Facial tissue thickness of american Caucasods. Maxwell Museum of Anthropology, Albuquerque, New Mexico, 1982.

[35] J.S. Rhine and H.R. Campbell. Thickness of facial tissues in american blacks. Journal of Forensic Sciences, 25(4):847-858, 1980.

[36] J. Sethian. Level Set Methods and Fast Marching Methods: Evolving Interfaces in Computational Geometry, Fluid Mechanics, Computer Vision and Materials Science. Cambridge University Press, 1999.

[37] J. Sethian. Level sets methods and fast marching methods. Cambridge University Press, 1999. 2nd Edition.

[38] R.W. Shahrom, P. Vanezis, R.C. Chapman, et al. Techniques in facial identification: computeraided facial reconstruction using laser scanner and videosuperimposition. Int. J. Legal Med., 108:194-200, 1996.

[39] V. Surazhsky, T. Surazhsky, D. Kirsanov, et al. Fast exact and approximate geodesics on meshes. In Proceedings of ACM SIGGRAPH 2005, volume 24, 2005.

[40] F. Tilotta, S. Gey, J. Glaunès, F. Richard, S. Verdeille, J.F. Gaudy, and Y. Rozenholc. Statistical facial reconstruction by tree functional regression on surfaces. In Proc. of the third Mediterranean Academy of Forensic Sciences Congress, Porto, Portugal, june 2007.

[41] P. Tu, R.I. Hartley, R.E. Lorensen, et al. Computer Graphic Facial Reconstruction, chapter 8: Face reconstruction using flesh deformation modes, pages 145-162. Academic Press, 2005.

[42] A.G. Tyrell, M.P. Evison, A.T. Chamberlain, and M.A. Green. Forensic three-dimensionnal facial reconstruction: historical review and contemporary developments. Journal of Forensic Science, 42(4):653-661, 1997.

[43] D. Vandermeulen, P. Claes, D. Loeckx, et al. Computerized craniofacial reconstruction using ct-derived implicit surface representations. Forensic Science International, 159S:164-174, 2006.

[44] P. Vanezis, R.W. Blowes, A.D. Linney, et al. Application of 3-D computer graphics for facial reconstruction and comparison with sculpting techniques. Forensic Science International, 42:69-84, 1989.

[45] P. Vanezis, M. Vanezis, G. McCombe, and T. Niblett. Facial reconstruction using 3-D computer graphics. Forensic Science International, 108:81-95, 2000. 
[46] R.H. Welcker. Schillers Schdel und todenmaske, nebst mittheilungen ber Schadel und todenmaske Kants. Fr. Vieweg und Sohn, Braunschweig, 1883.

[47] A. Yezzi, S. Kichenassamy, A. Kumar, et al. Geometric snakes for segmentation of medical imagery. IEEE Transaction on Pattern Analysis and Machine Intelligence, 16(2):199-209, 1997.

[48] Y. Zhang, M. Brady, and S. Smith. Segmentation of brain MR images through a hidden markov random field model and the expectation maximization algorithm. IEEE Transaction of Medical Imaging, 20(1):45-57, 2001.

\section{Appendix A. Description of ANATOMical POINTS}

The anatomical points are described in Tables 6 and 7 .

\begin{tabular}{|c|c|}
\hline Name & Description \\
\hline Vertex & $\begin{array}{l}\text { Highest ectocranial point on the skull's } \\
\text { midline when skull is in Frankfurt. }\end{array}$ \\
\hline Glabella & $\begin{array}{l}\text { Most anterior midline point on the frontal bone, } \\
\text { usually above the nasofrontal suture. }\end{array}$ \\
\hline Nasion & $\begin{array}{l}\text { Midline point where the two nasal bones } \\
\text { and the frontal intersect. }\end{array}$ \\
\hline Nasale & Midline point at the inferior free \\
\hline Rhinion & end of the internasal suture. \\
\hline Nasospinale & $\begin{array}{l}\text { Point where a line tangent to the most } \\
\text { inferior points of the two curves of the inferior } \\
\text { nasal aperture margin crosses the midline. }\end{array}$ \\
\hline Anterior & Pointed bony process at the \\
\hline nasal spine & inferior margin of the nasal aperture. \\
\hline Point A & $\begin{array}{l}\text { Deepest point in the bony concavity in the } \\
\text { midline below the anterior nasal spine, in the } \\
\text { region of the incisor roots. }\end{array}$ \\
\hline Prosthion & $\begin{array}{l}\text { Midline point at the most anterior point } \\
\text { on the alveolar process of the maxilla. }\end{array}$ \\
\hline Incisor superius & $\begin{array}{l}\text { Most anterior midline point on the } \\
\text { incisor crown. }\end{array}$ \\
\hline Infradentale & $\begin{array}{l}\text { Midline point at the superior tip of } \\
\text { the septum between the mandibular central incisors. }\end{array}$ \\
\hline Point B & $\begin{array}{l}\text { Deepest point in the profile curvature of the } \\
\text { mandible, from pogonion on the chin to infradentale. }\end{array}$ \\
\hline Pogonion & $\begin{array}{l}\text { Most anterior midline point on the chin } \\
\text { of the mandible. }\end{array}$ \\
\hline Gnathion & Most inferior midline point on the mandible. \\
\hline
\end{tabular}

TABLE 6. Description of even-numbered anatomical landmarks 


\begin{tabular}{|c|c|}
\hline Name & Description \\
\hline Mentale & $\begin{array}{l}\text { Most inferior point of the margin of } \\
\text { the mandibular mental foramen. }\end{array}$ \\
\hline Gonion & $\begin{array}{l}\text { Point along the rounded posteroinferior } \\
\text { corner of the mandible. }\end{array}$ \\
\hline Canine & $\begin{array}{l}\text { Most lateral point on the outer surface } \\
\text { of the canine. }\end{array}$ \\
\hline Ectomolare & $\begin{array}{l}\text { Most lateral point on the outer surface } \\
\text { of the alveolar margin of the maxilla, } \\
\text { often at the second molar position. }\end{array}$ \\
\hline Alare & $\begin{array}{l}\text { Most lateral point on the margin } \\
\text { of the nasal aperture. }\end{array}$ \\
\hline Infraorbitaire & $\begin{array}{l}\text { Most inferior point of the margin } \\
\text { of the infraorbitaire foramen. }\end{array}$ \\
\hline Condylion & Most lateral point \\
\hline laterale & on the mandibular condyle. \\
\hline Orbitale & Lowest point on the orbital margin \\
\hline Zygion & $\begin{array}{l}\text { Point of maximum lateral extent of the } \\
\text { lateral surface of the zygomatic arch. }\end{array}$ \\
\hline Jugale & $\begin{array}{l}\text { Point in the depth of the notch between the } \\
\text { temporal and frontal processes of the zygomatc bone. }\end{array}$ \\
\hline Ectoconchion & Most lateral point on the orbital margin. \\
\hline Orbitale superius & Highest point on the orbital margin. \\
\hline Euryon & Ectocranial point of greatest cranial breadth. \\
\hline
\end{tabular}

\section{Appendix B. Meshing ALGORIthm}

Let $\left\{A(i), i=1, \cdots, n_{A}\right\}$ and $\left\{B(j), j=1, \ldots, n_{B}\right\}$ be two lists of points decribing two successive curves. We fix $A(1)$ and $B(1)$ in such a way that

$$
d(A(1), B(1))=\min _{i, j} d(A(i), B(j)),
$$

where $d$ is the euclidian distance in $\mathbb{R}^{3}$. By convention, we let $A\left(n_{A}+1\right)=A(1)$ and $B\left(n_{B}+1\right)=B(1)$. We denote by $\operatorname{surf}(A, B, C)$ the triangle formed by the vertices $A, B$, and $C$. For all $(i, j)$, we compute

$$
S(i, j, 1)=\operatorname{surf}(A(i), A(i+1), B(j)) \text { and } S(i, j, 2)=\operatorname{surf}(A(i), B(j), B(j+1)) .
$$

The minimal cumulative surface we look for between points $(A(1), B(1)$ to $(A(j), B(j))$ is composed of a $k$ cumulated triangles denoted by $W_{k}(i, j)$. We further denote by $X_{k}(i, j)$ the indicator that the ancestor of segment $(A(i), B(j))$ on the minimal surface with $k$ triangles is $A(i-1)$ or $B(j-1)$ : if $\operatorname{surf}(A(i-1), A(i), B(j))$ belongs to the minimal cumulative surface $W_{k}(i, j)$ then $X_{k}(i, j)=1$ otherwise $X_{k}(i, j)=2$. We add the following condition: if $i+j-2>k$ or $i>n_{A}$ or $j>n_{B}$ then $W_{k}(i, j)=+\infty$.

The algorithm works now as follow :

- Set $W_{1}(1,1)=0$. 
- for $k=2, \ldots, n_{A}+n_{B}$ and for $i+j=k$, define

$$
\begin{aligned}
W_{k}(i, j)= & \min \left(W_{k-1}(i-1, j)+\operatorname{surf}(A(i-1), A(i), B(j)),\right. \\
& \left.W_{k-1}(i, j-1)+\operatorname{surf}(A(i), B(j), B(j-1))\right) .
\end{aligned}
$$

and set $X_{k}(i, j)=A(i-1)$, if the previous minimum is reached with the first term and $B(j-1)$ otherwise.

- Fix $i=n_{A}, j=n_{B}$,

- Create an empty list of triangle $L$,

- Repeat until $W_{i+j}(i, j)=0$ :

if $W_{k}(i, j)=1$, add to list $L$ triangle $(A(i-1), A(i), B(j))$ and change $i$ in $i-1$ otherwise add triangle $(A(i), B(j), B(j-1))$ and change $j$ in $j-1$.

- Return the list of triangles $L$ which is the minimal cumulative meshed surface. 